\title{
Prevalência da infecção por HIV em parturientes de maternidades vinculadas ao SUS*
}

\author{
Prevalence of infection with in unified health care system maternities
}

\author{
Lígia Mara Dolce de Lemos ${ }^{1}$, Ricardo Queiroz Gurgel ${ }^{2}$, Amaury Lelis Dal Fabbro ${ }^{3}$
}

\section{RESUM0}

\begin{abstract}
Objetivo: avaliar a prevalência do HIV nas parturientes de maternidades vinculadas ao SUS, pela utilização do teste rápido. Métodos: estudo transversal realizado em maternidades conveniadas ao SUS no Estado de Sergipe, após treinamento realizado com os profissionais de saúde dessas maternidades. As parturientes foram submetidas ao teste rápido imunocromatográfico para HIV, independente de já o terem realizado no pré-natal, após aconselhamento e assinatura do termo de consentimento livre e esclarecido. O teste utilizado foi Determine ${ }^{\mathrm{TM}}$ - Abbott Laboratórios do Brasil. Utilizou-se o esquema de profilaxia da transmissão vertical para todos os casos, segundo o protocolo recomendado pelo Ministério da Saúde. Foi criado banco de dados no Epi-Info 2002 e calculada a prevalência em relação a todos os testes realizados no período de janeiro de 2003 a março de 2004, por meio de análise estatística descritiva. Resultado: após realização de 9.215 testes rápidos, foram detectadas $39(0,42 \%)$ soropositivas para HIV, das quais $23(59 \%)$ não conheciam a sua soropositividade. Duas gestantes que já eram sabidamente HIV positivas não relataram aos profissionais sua condição no momento da admissão. O número de parturientes que referiram ter freqüentado o serviço de pré-natal foi alto ( $89 \%$ ), mas somente $32,5 \%$ destas foram submetidas ao teste para HIV na gestação. Conclusão: a prevalência detectada $(0,42 \%)$ neste estudo é semelhante à nacional. É alta a prevalência de parturientes que desconhecem sua soropositividade para HIV, o que indica inadequado funcionamento de cuidados pré-natais dispensados. Na situação atual é necessária a triagem no momento do parto, para garantia de intervenções adequadas neste momento crucial da transmissão do HIV.
\end{abstract}

PALAVRAS-CHAVE: Infecções na gravidez; Teste rápido para HIV; Transmissão vertical; AIDS

\section{ABSTRACT}

Purpose: to evaluate the prevalence of HIV infection in parturient women from maternity hospitals linked to the Public Health Service in Sergipe state (Brazil) using the rapid test, and to evaluate this strategy to introduce the protocol for adequate management of HIV patients. Methods: cross-sectional study which included the training of all health personnel and enrollment of every parturient woman admitted to maternity hospitals linked to the Public Health Service of Sergipe in the Northeast of Brazil. They received instructions about HIV perinatal transmission, were asked to undertake the rapid test, independently of their knowledge about their HIV serologic status, and signed the term of free consent. Additional information about antenatal care and obstetric history was obtained. The test used was the Determine ${ }^{\mathrm{TM}}-$ Abbott Laboratories, and we used the Health Ministry protocol for HIV infeccion prophylaxis. A database was created at Epi-Info 2002, and the prevalence was calculated in relation to all tests performed from January 2003 to March 2004. Results: after 9215 performed tests, 39 HIV-seropositive patients $(0.42 \%)$ were detected. Twenty-three of them (59\%) had no previous knowledge about their seropositivity. Two patients that already knew their positive sorology status did not refer this condition to the health persomel. The Health Ministry protocol was used for all positive cases. Antenatal care attendance was high (89\%) but only $32.5 \%$ of the patients had already been submitted to an HIV serologic test during pregnancy. Conclusions: HIV prevalence in pregnant women $(0.42 \%)$ in Sergipe was similar to the average rate in Brazil. A large number of seropositive pregnant women did not know their serologic status at the moment of delivery. This indicates bad antenatal care performance and the necessity to keep using HIV rapid test at the moment of labor and delivery in order to reduce vertical transmission.

KEYWORDS: Infection in pregnancy; HIV rapid test; Vertical transmission; AIDS

\footnotetext{
Maternidade Hildete Falcão Baptista - Aracaju-SE; Maternidade João Firpo - Aracaju - SE; Maternidade São José - Itabaiana - SE

1 Programa de Pós-Graduação em Ciências da Saúde da Universidade Federal de Sergipe

2 Coordenador do Núcleo de Pós-Graduação em Medicina da Universidade Federal de Sergipe

3 Departamento de Medicina Social da Faculdade de Medicina de Ribeirão Preto da Universidade de São Paulo

Correspondência: Lígia Mara Dolce de Lemos

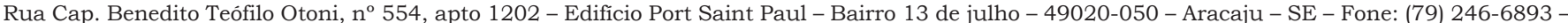
(79) 9982-0617 - e-mail: ligialemos@infonet.com.br

Apoio e financiamento pelo Centro de Estudos em Aids de Porto Alegre (CEARGS).

Recebido em: 3/8/2004 Aceito com modificações em: 22/12/2004
} 
Introdução

No inicio dos anos 90 a freqüência de casos de AIDS entre mulheres cresceu consideravelmente quando a transmissão heterossexual passou a ser a principal via de contágio ${ }^{1}$. A faixa etária que concentra os maiores percentuais de casos de AIDS em mulheres é a de 25 a 34 anos $^{2}$, levando ao aumento da transmissão vertical, pois estão em plena idade reprodutiva. Atualmente a quase totalidade de casos de AIDS em menores de 13 anos de idade tem como fonte de infecção a transmissão vertical do $\mathrm{HIV}^{3}$.

A probabilidade de transmissão vertical do HIV já está estabelecida, sendo evidenciado que a maioria dos casos de transmissão ocorre durante o trabalho de parto e no parto propriamente dito ${ }^{4-6}$, e que o aleitamento materno representa risco adicional de transmissão, de 7 a $22 \%^{7,8}$.

A partir de 1994, com a publicação dos resultados do protocolo Aids Clinical Trial Group (ACTG 076), surgiu a possibilidade de se estruturar intervenção eficaz e específica para a redução da transmissão vertical do $\mathrm{HIV}^{9}$, com recomendações para o uso do AZT no binômio mãe e filho ${ }^{10-12}$.

Embora haja no Brasil disponibilidade de testes e medicação profilática, as crianças continuam a ser infectadas ${ }^{2,13}$. Isso se deve ao fato de que muitas mulheres chegam às maternidades sem terem freqüentado o pré-natal e ao fato de a cobertura do teste para a infecção pelo HIV durante o pré-natal estar abaixo de $40 \%$ no Brasil $^{3,14}$ e $2 \%$ em Sergipe ${ }^{3,15}$. Isto significa que, para a maioria das mulheres soropositivas para HIV a única oportunidade de terem acesso ao aconselhamento, ao teste para a pesquisa do HIV e ao tratamento quimioprofilático da transmissão vertical é por ocasião da internação para o parto.

Apesar de não ser o momento ideal, não se deve negar à mulher a oportunidade de ter acesso ao diagnóstico e de fornecer o tratamento adequado ao recém-nascido, estando tal terapia disponível e podendo reduzir significativamente a chance de a criança se infectar pelo HIV.

Uma das estratégias de ampliar o acesso das gestantes ao diagnóstico da infecção pelo HIV foi o Projeto Nascer, lançado pelo Ministério da Saúde, cuja ação principal é o aconselhamento e testagem rápida para HIV nas parturientes que não tivessem sido avaliadas durante o pré-natal ${ }^{16}$.

O teste rápido é um ensaio imunocromatográfico que se utiliza para a detecção qualitativa de anticorpos HIV-1 e HIV-2 em sangue total, soro ou plasma humano, com facilidade de apresentar o diagnóstico em até 15 minutos, com simples coleta e fácil interpretação.
Diversos estudos foram realizados para avaliar o desempenho do teste rápido, comprovando sensibilidade e especificidade que o credenciam como recurso extremamente importante na indicação de medidas profiláticas que reduzam a transmissão perinatal deste vírus ${ }^{17,18}$. Outros trabalhos analisaram custo-beneficio de um programa para redução da transmissão vertical ${ }^{19,20}$.

Este estudo objetivou analisar a prevalência do HIV em parturientes de Sergipe, pelo teste rápido para detecção deste vírus no momento do parto.

\section{Pacientes e Métodos}

Foi realizado estudo transversal em três maternidades de Sergipe, sendo duas em Aracaju e uma em Itabaiana. A escolha destas maternidades se deu pelo fato de, juntas, serem responsáveis por aproximadamente metade dos partos no Estado. Em 2003 ocorreram em Sergipe 37.942 partos $^{21}$. Este projeto foi aprovado pelo Comitê de Ética em Pesquisa em Seres Humanos da Universidade Federal de Sergipe.

Todos os profissionais que trabalhavam nas maternidades selecionadas foram capacitados, priorizando os que atuam no pré-parto, sala de parto, puerpério e berçário, reforçando temas como acolhimento, aconselhamento pré e pós-teste, manejo clínico da gestante HIV positiva e seu concepto, biosseguranca, vigilância epidemiológica e realização do teste rápido, que foi demonstrado na prática, e a importância de obtenção do consentimento por escrito da parturiente. As condutas e coleta do teste rápido foram incorporadas à rotina de trabalho desses serviços.

De janeiro de 2003 até março de 2004, foram abordadas todas as parturientes admitidas nas maternidades selecionadas. Na admissão, recebiam aconselhamento pré- teste e com a anuência em participarem do estudo, eram submetidas ao teste rápido, independente de já terem sido ou não submetidas à avaliação sorológica no pré-natal.

Foi utilizado como triagem, o teste rápido para HIV Determine ${ }^{\mathrm{TM}}$ (Abbott Laboratórios), colhendo-se amostra de sangue total por meio de punção digital, com leitura visual para detecção qualitativa de anticorpos do HIV-1 e HIV-2, que produz resultados em até quinze minutos. Este teste possui uma sensibilidade de $100 \%$ e especificidade de 99,98\% para os anticorpos do HIV. Os kits são compostos de 10 tiras, que devem ficar a uma temperatura entre dois e $30^{\circ} \mathrm{C}$. 
Nas maternidades participantes do estudo foram selecionadas salas que se prestaram para o aconselhamento individual (SAT - sala de aconselhamento e testagem) e na impossibilidade de a gestante deambular, o exame era feito no próprio leito do pré-parto.

As parturientes cujo teste rápido foi reagente foram submetidas a exames confirmatórios segundo algoritmo do Ministério da Saúde

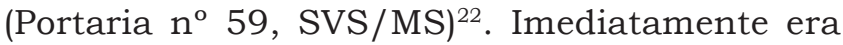
aplicado o protocolo ACTG $076^{10}$, além da entrega de leite para o recém-nascido.

Todos os dados destas parturientes foram anotados em livros específicos na sala de pré-parto, contendo informações como idade, sexo, realização de pré-natal, sorologia anti-HIV no pré-natal e resultados do teste rápido na maternidade. No cartão da gestante somente era anotado o tipo de teste realizado, sem o resultado, para garantir o sigilo por parte do serviço. No prontuário, foi confeccionado carimbo contendo as seguintes informações: realizado teste rápido para HIV com consentimento da paciente e o resultado - reagente/ não reagente.

Os insumos utilizados, como teste rápido para o HIV, inibidor de lactação, AZT injetável e xarope e fórmula infantil foram fornecidos pelo Ministério da Saúde.

Os dados foram digitados utilizando o programa Epi-Info e realizada análise estatística descritiva para cálculo de prevalência.

\section{Resultados}

Foram abordadas 9640 parturientes. A faixa etária de 20 a 29 anos prevaleceu. Quanto ao prénatal, 8601 gestantes $(89,2 \%)$ o freqüentaram, das quais $5572(57,8 \%)$ compareceram a seis ou mais consultas; no entanto, apenas $3136(32,5 \%)$ foram testadas para HIV no pré-natal (Tabela 1).

Nesta amostra, $327(3,4 \%)$ pacientes se negaram a participar do estudo. Desta forma, 9215 parturientes foram submetidas ao teste rápido para HIV nas três maternidades de Sergipe. A prevalência de parturientes com teste rápido reagente foi de $0,42 \%$ (39 casos) (Tabela 2 ).

Entre as parturientes com teste rápido regente, dezesseis eram acompanhadas no serviço de referência para DST/AIDS. Deste último grupo, duas parturientes omitiram sua condição no momento do internamento, porém, após o aconselhamento, concordaram em realizar o teste rápido, pelo qual se detectou a sua soropositividade.
Tabela 1 - Distribuição das parturientes segundo idade, assistência pré-natal, número de consultas e teste para HIV no pré-natal.

\begin{tabular}{|c|c|c|}
\hline \multirow[b]{3}{*}{ Variáveis } & \multicolumn{2}{|c|}{ População estudada } \\
\hline & $\mathrm{n}$ & $\%$ \\
\hline & 9640 & 100,0 \\
\hline \multicolumn{3}{|l|}{ Faixa etária } \\
\hline $10-19$ & 2256 & 23,5 \\
\hline $20-29$ & 4785 & 49,6 \\
\hline $30-39$ & 1684 & 17,4 \\
\hline $40 e+$ & 176 & 1,9 \\
\hline Ignorado & 739 & 7,6 \\
\hline \multicolumn{3}{|l|}{ Pré-natal } \\
\hline Sim & 8601 & 89,2 \\
\hline Não & 219 & 2,3 \\
\hline Ignorado & 820 & 8,5 \\
\hline \multicolumn{3}{|c|}{ Número de consultas } \\
\hline$<6$ & 4058 & 42,1 \\
\hline$\geq 6$ & 5572 & 57,8 \\
\hline Ignorada & 10 & 0,1 \\
\hline \multicolumn{3}{|c|}{ Teste para HIV no PN } \\
\hline Sim & 3136 & 32,5 \\
\hline Não & 5990 & 62,1 \\
\hline Ignorado & 514 & 5,4 \\
\hline
\end{tabular}

Tabela 2 - Distribuição das parturientes segundo realização do teste rápido para HIV na maternidade.

Parturientes

\begin{tabular}{lrr} 
Teste para HIV na maternidade & \multicolumn{1}{c}{ n } & \multicolumn{1}{c}{$\%$} \\
\hline Reagente & 39 & 0,4 \\
Não reagente & 9176 & 95,1 \\
Recusou & 327 & 3,3 \\
Ignorado & 98 & 1,0 \\
Total & 9640 & 100,0
\end{tabular}

Ao se compararem os casos com resultado positivo do teste rápido na maternidade em relação à freqüência ao pré-natal, observamos que $32(82,1 \%)$ das reagentes freqüentaram o prénatal, porcentagem semelhante à das não reagentes (Tabela 3$)$.

\section{Discussão}

Nas 9215 parturientes testadas, a prevalência de teste rápido reagente foi de $0,42 \%$, cifra semelhante à observada em outro estudo nacional ${ }^{3}$. 
Tabela 3 - Distribuição de parturientes segundo pré-natal e teste rápido para HIV na maternidade.

\begin{tabular}{|c|c|c|c|c|c|c|c|c|}
\hline \multirow{3}{*}{ Pré-natal } & \multicolumn{6}{|c|}{ Teste para HIV na Maternidade } & & \\
\hline & \multicolumn{2}{|c|}{ Reagente } & \multicolumn{2}{|c|}{ Não reagente } & \multicolumn{2}{|c|}{ Recusou } & \multicolumn{2}{|c|}{ Total } \\
\hline & $\mathbf{n}$ & $\%$ & $\mathbf{n}$ & $\%$ & $n$ & $\%$ & $\mathrm{n}$ & $\%$ \\
\hline Realizado & 32 & 82,1 & 8192 & 89,3 & 296 & 90,5 & 8601 & 89,2 \\
\hline Não realizado & 4 & 10,3 & 212 & & 3 & 0,9 & 219 & 2 \\
\hline Ignorado & 3 & 7,7 & 712 & 8,4 & 28 & 8,5 & 820 & 8 , \\
\hline Total & 39 & 100,0 & 9176 & 100,0 & 327 & 100,0 & 9640 & 100,0 \\
\hline
\end{tabular}

A epidemia do HIV vem avançando progressivamente na população feminina; além dos efeitos negativos sobre esta população, traz consigo a possibilidade da transmissão vertical do HIV, evento duplamente maléfico. Neste contexto, preocupa especialmente a relação verificada entre baixa escolaridade e não-recebimento de cuidados no prénatal, uma vez que esta última condição inviabiliza a realização da maior parte das intervenções para reduzir a transmissão vertical do $\mathrm{HIV}^{14}$.

O início tardio do pré-natal, após o primeiro trimestre da gestação, também se apresenta como barreira para a prevenção da transmissão verti$\mathrm{cal}^{23}$. No presente estudo, observamos que $58,8 \%$ tinham seis ou mais consultas de pré-natal, mas não foi avaliado o período da gestação em que se iniciou o pré-natal. Nesta situação, muitas vezes não se consegue realizar o diagnóstico antes do parto e, mesmo quando este é realizado, as intervenções disponíveis não são tão eficazes quanto as iniciadas mais precocemente na gestação. Desta forma, melhorar a cobertura e captar precocemente as gestantes para pré-natal de qualidade é fundamental para instituir, em tempo hábil, medidas de prevenção da transmissão vertical do HIV.

No município de Aracaju, uma das estratégias foi tornar obrigatório, mediante portaria, o oferecimento da sorologia para HIV nas consultas de pré-natal. Outras ações foram capacitações e melhoria do acesso aos exames pré-natais, e como resultado, observamos um aumento na confecção destes testes na gestação, mas com percentuais ainda muito baixos (32,5\%).

Neste estudo houve pequeno número de recusas $(3,3 \%)$ para realização do teste rápido nas maternidades, porém, todas as mulheres reagentes para HIV aceitaram a medicação profilática prescrita.

Os dados aqui apresentados sugerem que o baixo número de gestantes tratadas está associado à dificuldade de identificar a gestante infectada pelo HIV durante o pré-natal em Sergipe. Neste sentido, pelo menos dois fatores podem estar relacionados a esta dificuldade: a qualidade da assis- tência prestada à mulher durante o pré-natal, gestação e parto, e o acesso aos testes para HIV.

Entre os principais fatores que dificultam a identificação das gestantes no pré-natal e no momento do parto, pode-se destacar o não-oferecimento do teste para HIV durante a gestação por inadequada qualidade da informação por parte da equipe pré-natalista ${ }^{23}$. Prevalece, ainda, o desconhecimento da recomendação de oferecer o teste para HIV a todas as gestantes. Avaliação do próprio Ministério da Saúde evidencia a necessidade de capacitação da equipe de saúde para o aconselhamento pré e pós-teste ${ }^{24}$. Como se pouco fosse, as condições socioeconômicas favorecem a desinformação das gestantes sobre a importância do teste e comprometem a percepção do risco da infecção pelo HIV ${ }^{25}$. Destaquem-se ainda os problemas para efetivação do teste e obtenção dos resultados após a solicitação pelo profissional de saúde, por conta de disponibilidade insuficiente de exames na rede, centralização excessiva da realização da sorologia e problemas na logística de envio de amostras para o laboratório e de resultados para os serviços de pré-natal.

A necessidade de ampliar o acesso das gestantes ao diagnóstico da infecção pelo HIV requer que diferentes iniciativas sejam tomadas para aumentar progressivamente a descentralização do teste e a disponibilização de exames.

A decisão de se fazer o teste rápido em todas as parturientes, independente de já terem feito a sorologia no pré-natal, mostrou-se relevante, pois algumas com teste negativo para HIV, realizado no período pré-natal, apresentaram-se reagentes no momento do parto por meio do teste rápido. Nestes casos poderiam estar em período de "janela imunológica" ou mesmo terem se infectado após a realização do mesmo e perderiam a oportunidade de se enquadrar no protocolo estabelecido.

Neste contexto, os novos testes rápidos para o diagnóstico da infecção pelo HIV certamente são instrumentos de grande utilidade para a redução da transmissão vertical do HIV, principalmente devido ao fato de que o período de maior transmissão (entre 50 e $70 \%$ ) ocorre próximo ao parto ou durante o mesmo ${ }^{5,6,9}$.

Este trabalho com teste rápido para HIV no momento do parto permitiu cobertura de $96,5 \%$ das parturientes das maternidades selecionadas e também evidenciou o percentual de gestantes que na época do início do estudo conheciam o seu status sorológico para HIV ainda no pré-natal $(32,5 \%)$. Com essa estratégia foi possivel detectar 23 casos que desconheciam sua soropositividade, além de outras duas pacientes que, mesmo sabendo de sua condição sorológica, não a referiram no momento 
do parto. Propõe-se disponibilizar universalmente o exame com o teste rápido para todas as parturientes que não tiverem consigo o resultado de uma sorologia para HIV no terceiro trimestre da gestação.

\section{Referências}

1. World Health Organization. Joint United Nations Program on HIV / AIDS (UNAIDS). 2001 AIDS epidemic update. Geneva: WHO; 2002.

2. Boletim Epidemiológico AIDS. Ministério da Saúde. Coordenação Nacional de DST e AIDS. Brasília 2003; 17(1).

3. Boletim Epidemiológico AIDS. Ministério da Saúde. Coordenação Nacional de DST e AIDS. Brasília 2002; 16(1).

4. Mofenson LM, Lambert JS, Stiehm ER, et al. Risk factors for perinatal transmission of human immunodeficiency virus type 1 in women treated with zidovudine. N Engl J Med. 1999; 341:385-93.

5. Rogers MF, Ou CY, Rayfield M, et al. Use of the polymerase chain reaction for early detection of the proviral sequences of human immunodeficiency virus in infants born to seropositive mothers. N Engl J Med. 1989; 320:1649-54.

6. Rouzioux C, Costagliola D, Burgard M, et al. Timing of mother-to-child HIV-1 transmission depends on maternal status. AIDS 1993; 7 (Suppl 2):S49-52.

7. Bertoli J, St Louis ME, Simonds RJ, et al. Estimating the timing of mother-to-child transmission of human immunodeficiency virus in a breast-feeding population in Kinshasa, Zaire. J Infect Dis. 1996; 174:722-6.

8. Ekpini ER, Wiktor SZ, Satten GA, et al. Late postnatal mother-to-child transmission of HIV-1 in Abidjan, Cote d'Ivoire. Lancet. 1997; 349:1054-9.

9. Connor EM, Sperling RS, Gelber R, et al. Reduction of maternal-infant transmission of human immunodeficiency virus type 1 with zidovudine treatment. Pediatric AIDS Clinical Trial Group Protocol 076 Study Group. N Engl J Med. 1994; 331:1173-80.

10.Recommendations of the U.S. Public Health Service Task Force on the use of zidovudine to reduce perinatal transmission of human immunodeficiency virus. MMWR Recomm Rep. 1994; 43:1-20.

11.Administration of zidovudine during late pregnancy and delivery to prevent perinatal HIV transmissionThailand. MMWR Morb Wkly Rep. 1998; 47:151-4.

12.Ministério da Saúde. Coordenação Nacional de DST/ AIDS. Recomendações para profilaxia da transmissão materno-infantil do HIV e terapia antiretroviral em gestantes. Manual. Brasília: Coordenação Nacional DST/AIDS; 2002.

13.Tess BH, Rodrigues LC, Newell ML, Dunn DT, Lago TD. Breastfeeding, genetic, obstetric and other risk factors associated with mother-to-child transmission of HIV-1 in São Paulo State, Brazil. São Paulo Collaborative Study for Vertical Transmission of HIV-1. AIDS 1998; 12:513-20.

14.Ministério da Saúde. Manual técnico de assistência pré-natal. Brasília; 2000.

15. Boletim epidemiológico. Secretaria Estadual de Saúde de Sergipe. Coordenação Estadual de DST/ AIDS. Sergipe 2002; set.-out.

16. Ministério da Saúde. Coordenação Nacional de DST/ AIDS. Projeto nascer- maternidades. Brasília; 2003.

17.Carvalho RL, Krahe C, Farina G, Paula DO, Richetti $\mathrm{N}$, Crossetti T. Teste rápido para diagnóstico da infecção pelo HIV em parturientes. Rev Bras Ginecol Obstet. 2004; 26:325-8.

18.Duarte G, Gonçalves CV, Marcolin AC, Paschoini MC, Quintana SM, Mussi-Pinhata MM. Teste Rápido para detecção pelo HIV-1 em gestantes. Rev Bras Ginecol Obstet. 2001; 23:107-11.

19.Grobman WA, Garcia PM. The cost-effectiveness of voluntary intrapartun rapid human immunodeficiency virus testing for women without adequate prenatal care. Am J Obstet. 1999; 181:1062-71.

20.Stringer JS, Rouse DJ. Rapid testing and zidovudine treatment to prevent vertical transmission of human immunodeficiency virus in unregistered parturients: a cost-effectiveness analysis. Obstet Gynecol. 1999; 94:34-40.

21. Ministério da Saúde. Sistema de informações de nascidos vivos (SINASC) [citado 2004 jul 20]. Disponivel em: URL: http://www.datasus.gov.br/ tabnet.htm.

22. Ministério da Saúde. Portaria técnica ministerial n.59, de 28 de janeiro de 2003. Diário Oficial da República Federativa do Brasil, Brasília (DF), 2003 jan 29; Sec.1:1-9.

23. Marques HHS, Latorre MRDO, DellaNegra M, Pluciennik AMA, Salomão MLM. Falhas na identificação da infecção pelo HIV durante a gravidez em São Paulo, SP, 1998. Rev Saúde Pública. 2002; 36:385-92.

24. Ministério da Saúde. Coordenação Nacional de DST/AIDS. Manual de aconselhamento: um desafio para prática integral: avaliação das ações. $1^{a}$ ed. Brasília; 1999.

25.Coimbra LC, Silva AAM, Mochel EG, et al. Fatores associados à inadequação do uso da assistência pré-natal. Rev Saúde Pública. 2003; 37:456-62. 